\title{
ACYCLICITY IN THREE-MANIFOLDS
}

\author{
BY D. R. MCMILLAN, JR. ${ }^{1}$
}

Abstract. An acyclic compactum in an orientable, open 3-manifold has arbitrarily close, polyhedral neighborhoods whose components are compact 3-manifolds with a special structure. Frequently, these 3-manifolds have free fundamental groups. These observations and some results from combinatorial group theory are exploited to deduce facts about the homomorphism of fundamental groups induced by an acyclic mapping. The techniques are applied to relate local homotopy properties of quotient spaces of acyclic upper semicontinuous decompositions, to "UV" (or "shape") properties of the elements in the decomposition. It is shown that a "0-dimensional" monotone decomposition of Euclidean $k$-space is acyclic if the quotient space is an open $k$-manifold. (For $k=3$, such a decomposition is shown to be cellular.) Some conditions are given under which acyclic decompositions are cellular.

1. Introduction. Let $G$ be an upper semicontinuous decomposition of Euclidean 3-space $E^{3}$, into compact, connected sets such that for some prime $p$, each $g \in G$ is strongly 1 -acyclic over $Z_{p}$ (the integers modulo $p$ ). Our purpose is to show that some useful information of a homotopy-theoretic nature about the decomposition space $E^{3} / G$ and the projection mapping $P_{G}$, can be deduced from an examination of the nondegenerate elements of $G$ (whose union is denoted $H_{G}$ ). For example, we prove that a necessary condition that $E^{3} / G$ should be locally simply connected at $P_{G}(g)$, is that $g$ should have property 1-UV. (These and other terms are defined later.)

In particular, if $X$ is a continuum in $E^{3}$ which is strongly 1-acyclic over $Z_{p}$, then $E^{3}$ "modulo" $X$ is locally simply connected if and only if $X$ has property 1-UV. This agrees with R. H. Bing's announcement [7] that $E^{3}$ modulo a solenoid is not simply connected and hence not locally simply connected. In light of the 1-dimensional continuum of

An address delivered to the Annual Meeting of the American Mathematical Society at San Antonio, Texas, on January 23, 1970, by invitation of the Committee to Select Hour Speakers for Summer and Annual Meetings; received by the editors April 9, 1970.

AMS 1969 subject classifications. Primary 5705; Secondary 5460, 5478, 6560, 5568.

Key words and phrases. Acyclicity, 3-manifold, handlebody, cube-with-handles, upper semicontinuous decomposition, equivalent decompositions, zero-dimensional decomposition, cellularity, UV properties, fundamental shape, free group, central series, local homotopy properties.

1 Research supported by the University of Wisconsin Graduate School and by grant NSF GP-16093. 
J. H. Case and R. E. Chamberlin in $\$ 5$ of [10], our result also eliminates the possibility that the more stringent hypothesis on $X$ of strong 1 -acyclicity over $Z$ (the integers) would yield a different answer. (The example of [10] is acyclic over every coefficient group, and hence we have answered the questions raised by $\mathrm{K}$. Borsuk in [8, Problem 3.3, p. 214], and by D. M. Hyman in [14, p. 67].) In the case of the specific example $M$ of [10], S. Armentrout has shown independently that $E^{3}$ modulo $M$ is not simply connected.

Our other main result concerns the case in which $G$ is a compact decomposition of $E^{3}$, each of whose elements is strongly acyclic. We prove that for each open, connected set $U \subset E^{3} / G$, the projection mapping from $P_{G}^{-1}(U)$ onto $U$ induces a monomorphism on fundamental groups. When this fact is combined with the result above, we obtain several necessary conditions for the existence of a homeomorphism $h: E^{3} / G \rightarrow E^{3} / F$ such that $h\left(P_{G} \bar{H}_{G}\right)=P_{F} \bar{H}_{F}$ (where $F$ is also a compact decomposition of $E^{3}$ ). Namely, if an element $g \in G$ "corresponds" under $h$ to an element $f \in F$, then: $f$ has property $\mathrm{UV}^{\infty}$ if and only if $g$ has property $\mathrm{UV}^{\infty}$; and $f$ is cellular if and only if $g$ is cellular. Further, it has been conjectured that $E^{3}$ modulo a compact set $X$ with property UV ${ }^{\infty}$ yields $E^{4}$ when multiplied by $E^{1}$. It follows from our results that "property UV' ${ }^{\infty}$ " is necessary for this conclusion, and that it suffices to prove the conjecture when $X$ is a treelike continuum.

A knowledge of the main results and terminology of [26] will be helpful. We now mention several conventions and definitions for later use. The symbol $Z_{p}$ ( $p$ always denotes 0 or a prime) is to be read consistently in a given discussion, with $Z_{0}=Z$ the infinite cyclic group. If $n$ is an integer, $n \geqq 1$, then a compact set $X \subset M$ is strongly $n$-acyclic over $Z_{p}$ (or "has property $n$-uv $\left(Z_{p}\right)$ ") if each open set $U \subset M$ containing $X$ contains an open set $V$ such that $X \subset V$ and such that each $n$-cycle in $V$ is homologous to zero in $U$ (singular homology, $Z_{p}$ coefficients). For example, the usual dyadic solenoid is strongly 1 -acyclic over $Z_{2}$, but not over $Z$, even though its integral Cech homology is zero. If $n \geqq 0$ and we replace, at each occurrence in our definition, " $n$-cycle" by "singular $n$-sphere" and "homologous to zero" by "homotopic to a constant," then we obtain the definition of property $n$-UV. The statement " $X \subset M$ has property $\mathrm{UV}^{n}$ " means that $X$ has property $i$-UV for $0 \leqq i \leqq n$. Thus, "strongly $n$-acyclic" is the homology analogue of property $n$-UV (see [6] and [26]). A compact set $X \subset M$ is strongly acyclic over $Z_{p}$ (or "has property $\mathrm{uv}^{\infty}\left(Z_{p}\right)$ ") if it is connected and is strongly $n$-acyclic over $Z_{p}$ for each $n \geqq 1$ (cf. the remarks in $[26, \S 3])$. The corresponding homotopy property is called property $\mathrm{UV} \infty$. Clearly, a compact subset $X$ of a manifold has 
property 0-UV if and only if $X$ is connected. We let $E^{n}, S^{n}$, and $\Delta^{n}$ denote, respectively, Euclidean $n$-space, the $n$-sphere, and the $n$ simplex. $T^{i}$ is the $i$-skeleton of a given triangulation $T$. As usual, manifolds are connected.

Many of our results are more general than stated above, and apply to 3-manifolds other than $E^{3}$. In general, we have stated our main results in terms of compact, monotone mappings, and our corollaries in the language of decompositions. See $[6, \S 6]$ for the connection. Many different authors have obtained results on UV properties and related topics (see $[2],[3],[6],[9],[14],[15],[16],[18],[19],[27]$, and $[30])$, and we have made no attempt to cite all the possible references for a given fact.

2. Some geometric-algebraic background. Recall that a mapping is said to be compact if the pre-image of each compact set is compact. A mapping is called monotone if each point-inverse is compact and connected. A mapping is a $U^{n}$ mapping if each of its point-inverses has property UVn.

In the context of the next theorem, the statement that a singular $n$-sphere in $X$ represents an element of $N$ means that the set of elements in $\pi_{n}(X)$ generated by the singular $n$-sphere under the action of $\pi_{1}(X)$ (see [31, p. 384]), is contained in $N$. Since $N$ is assumed to be invariant under the action of $\pi_{1}(X)$, this requirement is unambiguous. Note that if $n=1$, then we are just requiring that $N$ be normal in $\pi_{1}(X)$.

Theorem 1. Suppose that $X$ and $Y$ are pathwise-connected metric spaces, that $P$ is a compact $\mathrm{UV}^{n-1}$ mapping from $X$ onto $Y(n \geqq 1)$, and that $N$ is a subgroup of $G=\pi_{n}\left(X, x_{0}\right)$, with $N$ invariant under the action of $\pi_{1}(X)$. Suppose further that the following holds for each $y \in Y$ : there is an open set $W_{y} \subset X$ with compact closure, such that $P^{-1}(y) \subset W_{y}$ and such that each singular $n$-sphere in $W_{y}$, when considered in $X$, represents an element of $N$. Then the kernel of the homomorphism

$$
P_{*}: \pi_{n}\left(X, x_{0}\right) \rightarrow \pi_{n}\left(Y, P\left(x_{0}\right)\right)
$$

is contained in $N$.

Proof. Let $L=\partial \Delta^{n+1}$. Suppose that $g: \Delta^{n+1} \rightarrow Y$ and $f: L \rightarrow X$ are maps with $g \mid L=P f$. We wish to show that $f$ represents an element of $N$. Let $\epsilon$ be a positive number so small that if $A$ is a subset of $Y$ of diameter less than $\epsilon$ and if $A \cap g\left(\Delta^{n+1}\right) \neq \varnothing$, then $P^{-1}(A)$ is contained in some $W_{y}$. Let $T$ be a subdivision of $\Delta^{n+1}$ such that for each $i$-simplex $\sigma^{i} \in T, i \leqq n+1, g\left(\sigma^{i}\right)$ has diameter less than $\epsilon / 3$. Denote $T^{n}$, the $n$-skeleton of $T$, by $K$. 
According to [6, Lemma 3.2], there is an extension $F$ of $f$ sending $K$ into $X$ such that $D(g \mid K, P F)<\epsilon / 3$, where $D$ denotes the metric on $Y$. Clearly, for each $\sigma^{n+1} \in T, P F\left(\partial \sigma^{n+1}\right)$ has diameter less than $\epsilon$. Hence, $P^{-1} P F\left(\partial \sigma^{n+1}\right)$ is contained in some $W_{y}$. Thus, for each $\sigma^{n+1} \in T, F \mid \partial \sigma^{n+1}$ represents an element of $N$. It follows that $F \mid \partial \Delta^{n+1}$ $=f$ represents an element of $N$.

Following J. Stallings in [32], if $Q$ is a subgroup of the group $G$ and $p$ is zero or a prime, then we define $G \# Q$ to be the subgroup of $G$ generated by all elements

$$
g u g^{-1} u^{-1} v^{p} \text {, where } g \in G, \quad u, v \in Q .
$$

The (descending) central series of $G$ corresponding to $p$ is defined inductively by putting $G_{0}=G, G_{\alpha+1}=G \# G_{\alpha}$, and $G_{\beta}=\bigcap_{\alpha<\beta} G_{\alpha}$, if $\beta$ is a limit ordinal. Note that for $p=0$, this definition gives the lower central series of $G$, whose term $G_{1}$ is the commutator subgroup of $G$. Each $G_{\alpha}$ is fully invariant in $G$ (see $[21$, p. 74]), hence normal. Stallings has shown in [32, Theorem 6.3] that if $G$ is a free group, then for the first infinite ordinal $\omega, G_{\omega}=1$. If $G$ is a finitely-generated abelian group and $p \neq 0$, then $G_{\omega}$ consists of all elements whose orders are finite and relatively prime to $p$.

Now suppose that $X \subset M$ is a compact set which has property $\mathrm{UV}^{n-1}, n \geqq 1$, and is strongly $n$-acyclic over $Z_{p}$. It follows that $X$ satisfies the usual "UV" statement with $U$ and $V$ connected and the image $H_{n}(V ; Z) \rightarrow H_{n}(U ; Z)$ contained in the subgroup $p \cdot H_{n}(U ; Z)$. Hence, if $n=1$, we find that the image $\pi_{n}(V) \rightarrow \pi_{n}(U)$ is contained in the term $G_{1}$ of the $p$-central series for $G=\pi_{n}(U)$. For $n>1$, we can prove the same "UV" statement by the methods of $[19$, Theorem 4.2]. Hence:

Corollary 1.1. Suppose $M^{k}$ is a k-manifold, possibly with boundary, and that $P$ is a compact, $\mathrm{UV}^{n-1}$ mapping $(n \geqq 1)$ of $M^{k}$ onto a Hausdorff space $Y$. Suppose that for each $y \in Y, P^{-1}(y)$ is contained in a compact set $A_{y} \subset M^{k}$, such that $A_{y}$ is strongly n-acyclic over $Z_{p}$. Then the kernel of the homomorphism

$$
P_{*}: \pi_{n}\left(M^{k}, x_{0}\right) \rightarrow \pi_{n}\left(Y, P\left(x_{0}\right)\right)
$$

is contained in the term $G_{\omega}$ of the central series of $G=\pi_{n}\left(M^{k}, x_{0}\right)$ corresponding to $p$.

We remark that our corollary adds nothing new when $p=0, n>1$, and each $A_{y}=P^{-1}(y)$. For, R. C. Lacher shows in [19] in this case, that $P$ is a $\mathrm{UV}^{n}$ mapping and hence induces $\pi_{n}$-isomorphisms. If $p=0$ and $n=1$, then a stronger result than claimed can actually be de- 
duced. Namely, that kernel $P^{*}$ is contained in each of the successive derived subgroups of $G$ (see [21, p. 293]).

In the proof of Theorem 1 for $n=1$, we can replace $\Delta^{2}$ by an appropriate mapping cylinder of a map from $\partial \Delta^{2}$ to a wedge of circles, and obtain the following generalization. Details are left to the interested reader (see [21, p. 74$]$ for our notation and for a discussion of verbal subgroups).

Theorem 1'. Assume the hypotheses of Theorem 1 with $n=1$. Let $W_{\mu}\left(X_{\lambda}\right)$ be a set of words in the symbols $X_{\lambda}(\mu, \lambda=1,2, \cdots)$. Let $G=\pi_{1}\left(X, x_{0}\right), H=\pi_{1}\left(Y, P\left(x_{0}\right)\right)$, and $\alpha \in G$. If $P_{*}(\alpha)$ belongs to the $W_{\mu}$-verbal subgroup $H\left(W_{\mu}, \cdots\right)$, then $\alpha \in N \cdot G\left(W_{\mu}, \cdots\right)$. Hence, if $N \subset G\left(W_{\mu}, \cdots\right)$, then $P$ induces a monomorphism

$$
G / G\left(W_{\mu}, \cdots\right) \rightarrow H / H\left(W_{\mu}, \cdots\right) .
$$

REMARK. In the context of Corollary 1.1, one can obtain from Theorem 1', obvious "monomorphism" corollaries about the induced mappings between the quotient groups of $G=\pi_{1}\left(M^{n}\right)$ and $H=\pi_{1}(Y)$ by the corresponding terms in their central series corresponding to $p$.

We shall need later the following sharpened form of Theorem 2 of [26]. The proof of Theorem 2 relies on the Finiteness Theorem of W. Haken [12, p. 48]. However, it suffices for our purpose to know his result in the case where all the incompressible surfaces being considered are closed (i.e., compact and without boundary). Moreover, we need only a finite upper bound on the number of disjoint, incompressible, polyhedral surfaces which can exist in the compact 3-manifold $M^{3}$ with no two of them being parallel. That is, Haken's specific estimate of $61 \alpha$ does not matter to us.

Haken's argument simplifies considerably (and certain difficulties do not arise) when $M^{3}$ is irreducible and the surfaces are closed. The general result (for collections of closed surfaces) follows from the irreducible one and from the fact that $M^{3}$ can be cut along a collection of polyhedral 2-spheres, and 3-cells attached to the boundary of the resulting 3-manifold, so as to obtain an irreducible 3-manifold (see [12, p. 42]). This program is not hard to carry out, and is recorded in [35]. I have learned that Harry Row has independently obtained a proof along these lines.

THEOREM 2. Let $X$ be a compact, proper subset of Int $M^{3}$, where $M^{3}$ is an orientable piecewise-linear 3-manifold. Let $p$ denote 0 or a prime, and suppose that $X$ has the following property relative to $M^{3}$ and $p:$ For each open set $U \subset M^{3}$ with $X \subset U$, there is an open set $V, X \subset V \subset U$, such that under inclusion, 


$$
H_{1}\left(V-X ; Z_{p}\right) \rightarrow H_{1}\left(U ; Z_{p}\right)
$$

is zero. Then, $X=\bigcap_{i=1}^{\infty} H_{i}$, where $H_{i}$ is a compact polyhedron in $M^{3}$, each component of $H_{i}$ is an orientable 3-manifold with nonempty boundary, $H_{i+1} \subset$ Int $H_{i}$, and each component of $H_{i}$ has the following structure: it is obtained from a compact 3-manifold $Q^{3}$ whose boundary consists entirely of 2-spheres, by adding to $\partial Q^{3}$ a finite number of (solid) 1handles.

Proof. We emphasize that the manifold $Q^{3}$ is permitted to vary with the choice of $i$ and with the component of $H_{i}$ being considered. The designation $Q^{3}$ is only for convenience in later references. Our special hypothesis on $X$ is hereditary with respect to subsets of $X$ which are both open and closed in $X$. Hence, it suffices to show that $X$ has a neighborhood of the required type in $M^{3}$.

The proof is the same as the proof of [26, Theorem 2], except for the modifications which we indicate here. We retain the notation of [26], with $Z_{p}$ replacing $Z_{*}$. Note that we have had to assume orientability of $M^{3}$, whereas in [26] the hypotheses guaranteed an orientable neighborhood of $X$ in $M^{3}$. Also because of our weakened hypotheses, we are able to assume, as in [26], neither that $\partial M^{3}$ is connected nor that $M^{3}$ is separated by each polyhedral surface in $M^{3}$. Thus, 2spheres in $M^{3}$ not only may fail to bound homology cells, they may even fail to separate $M^{3}$. As before, we may assume that $M^{3}$ is compact, and the integer $H$ is selected in the same manner. Again, we consider nested, ordered $H$-tuples $\Sigma$ of $Z_{i}$ 's but require that they possess a characteristic property weaker than the one used in [26]. Namely, we insist only that each 1 -cycle in $\partial Z_{i}$ should "bound" in Int $Z_{i-1}$.

The rest of the proof is essentially unchanged. That is, we detect $Z_{m}^{*}$ in $\Sigma^{*}$ such that $\partial Z_{m}^{*}$ consists entirely of 2 -spheres. We make no attempt to put $Z_{m}^{*}$ in a $Z_{p}$-homology 3-cell $H_{0}^{3}$, but instead let $H_{0}^{3}\left(=Q^{3}\right)$ be obtained by joining efficiently the components of $Z_{m}^{*}$ by tubes, and then taking a regular neighborhood of the result. The proof is completed as before by invoking [26, Theorem 1$]$ to provide the required neighborhood of $X$ in $M^{3}$.

Corollary 2.1. Let $X$ be a compact, proper subset of Int $M^{3}$, where $M^{3}$ is a piecewise-linear 3-manifold and each component of $X$ is strongly 1-acyclic over $Z_{p}\left(p=0\right.$ or a prime). If $p>2$, assume also that $M^{3}$ is orientable. Then, $X=\bigcap_{i=1}^{\infty} H_{i}$, where $H_{i}$ is a compact polyhedron in $M^{3}$, each component of $H_{i}$ is an orientable 3-manifold with nonempty boundary, $H_{i+1} \subset \operatorname{Int} H_{i}$, and each component of $H_{i}$ has the following structure: it is obtained from a 3-manifold $Q^{3}$ with $H_{1}\left(Q^{3} ; Z_{p}\right)=0$, by adding to 
$\partial Q^{8}$ a finite number of (solid, orientable) 1-handles, none of which joins different components of $\partial Q^{3}$. If, in addition, each component of $X$ is strongly acyclic over $Z_{p}$, then each component of $H_{i}$ can be chosen to have connected boundary. If each component of $X$ also has property 2-UV, then each component of $H_{i}$ can be chosen to be a homotopy cube-withhandles.

Proof. We note the word of caution about $Q^{3}$ given at the start of the proof of Theorem 2. Again, it suffices to find a neighborhood of $X$ in $M^{3}$ of the required type. There is a 3-dimensional, orientable submanifold $M_{0}^{3}$ of $M^{3}$ with the inclusion $H_{1}\left(M_{0}^{3} ; Z_{p}\right) \rightarrow H_{1}\left(M^{3} ; Z_{p}\right)$ zero, so that (as is easily shown) each 2-sided polyhedral surface in $M_{0}^{3}$ separates $M_{0}^{3}$.

Clearly the hypotheses of Theorem 2 are met. Let $H_{1}, H_{2}, \cdots$ be the polyhedra provided by Theorem 2 , with $H_{1} \subset$ Int $M_{0}^{3}$. The 1-handles added to each $\partial Q^{3}$ (in the conclusion of Theorem 2) do not join up different components of $\partial Q^{3}$, because each (necessarily 2-sided) 2-sphere in $M_{0}^{3}$ separates $M_{0}^{3}$. To insure that each $H_{1}\left(Q^{3} ; Z_{p}\right)$ $=0$, select (and relabel) a subsequence of $H_{1}, H_{2}, \cdots$, with each inclusion $H_{i+1} \rightarrow H_{i}$ inducing the zero homomorphism on 1-dimensional $Z_{p}$-homology. The desired result then follows, since $\partial Q^{3}$ consists entirely of 2-spheres and hence (from a Mayer-Vietoris sequence) the inclusion $Q^{3} \rightarrow H_{i-1}$ induces a monomorphism on 1-dimensional $Z_{p}$-homology.

In case each component of $X$ is strongly acyclic over $Z_{p}$, we argue that the above sequence can be chosen so that each polyhedral 2sphere in $H_{i+1}$ bounds a $Z_{p}$-homology 3-cell in $H_{i}$. This fact is then used to replace $H_{i+1}$ by an $H_{i+1}^{*} \subset \operatorname{Int} H_{i}$, where $\partial H_{i+1}^{*}$ is connected. Details are left to the reader. For the last assertion, see the proof of Theorem 3 of [26].

REMARK 1. If $N^{3}$ is a compact 3-manifold whose boundary contains exactly $n 2$-spheres, $n \geqq 1$, then we can remove $(n-1)$ "tunnels" from $N^{3}$ so as to obtain a 3-manifold with the same fundamental group, but having boundary which contains exactly one 2 -sphere. Hence, the $Q^{3}$ of Theorem 2 and Corollary 2.1 can be expressed as $Q_{0}^{3}$ plus a finite number of disjoint 2-handles, where $\partial Q_{0}^{3}$ is a 2-sphere and $\pi_{1}\left(Q_{0}^{3}\right)$ is isomorphic to $\pi_{1}\left(Q^{3}\right)$. Thus,

$$
H_{1}\left(Q_{0}^{3} ; Z_{p}\right) \approx H_{1}\left(Q^{3} ; Z_{p}\right) .
$$

REMARK 2. Some pleasing geometric observations result from Corollary 2.1. Let $X$ and $M^{3}$ be as given there. Then, in order to show that each component of $X$ is strongly acyclic over $Z_{p}$, it suffices to 
check the "UV" definition of strong 2-acyclicity with respect to 2cycles which can be represented by polyhedrally embedded 2-spheres in $V$. Further, we need only show that such 2-cycles bound with $Z_{p^{-}}$ coefficients, even when proving that each component of $X$ is strongly acyclic over $Z$. Thus we can, for example, always replace "strongly acyclic over $Z_{p}$ and has property 2-UV" by "strongly 1-acyclic over $Z_{p}$ and has property $2-\mathrm{UV}$ " when considering a compact, connected set in an orientable, nonclosed 3-manifold. See Theorems 3 and 4 of [26] and their corollaries as cases in point.

For future reference, we collect in the following lemma some useful facts about strong acyclicity. The proofs are left to the reader. Part (i) is an easy compactness argument. Part (ii) uses the definition and symmetry of linking for two oriented "bounding" simple closed curves in an oriented $M^{3}$. The last two parts depend on Corollary 2.1 and its Remark 2. All parts assume it known that a compact, proper subset of the interior of $M^{3}$ has arbitrarily close, compact, polyhedral neighborhoods each of whose components is a compact 3-manifold with nonempty boundary.

Lemma 1. Let $X$ be a compact, proper subset of the interior of an orientable, piecewise-linear 3-manifold $M^{3}$. Let $p$ denote 0 or a prime. Then the following propositions hold.

(i) If $n$ is a positive integer, $G$ is an abelian group, and $\mathrm{R}$ is one of the properties " $n$-UV" or "strongly n-acyclic over $G$ " then $X$ has property $\mathrm{R}$ if and only if each component of $X$ has property $\mathrm{R}$.

(ii) $X$ is strongly 1-acyclic over $Z_{p}$ if and only if some open neighborhood $U$ of $X$ in $M^{3}$ has these properties: each polyhedral simple closed curve in $U$ (considered as a 1-cycle over $Z_{p}$ ) is $Z_{p}$-homologous to zero in $M^{8} ;$ and in the same sense, each polyhedral simple closed curve in $U-X$ is $Z_{p}$-homologous to zero in $M^{3}-X$. (In particular, this condition is met if $M^{3}$ is $E^{3}$ or $S^{3}$, and if $H_{1}\left(M^{3}-X ; Z_{p}\right)=0$.)

(iii) Suppose that $X$ is connected and strongly 1-acyclic over $Z_{p}$. Then $X$ is strongly acyclic over $Z_{p}$ if and only if some connected, open neighborhood $U$ of $X$ in $M^{3}$ has these properties: each polyhedral 2sphere in $U$ separates $U$; and for each $p, q \in U-X$ there is an open set $V, X \subset V \subset U$, such that no polyhedral 2-sphere in $V$ separates $p$ from $q$ in $U$.

(iv) Suppose that $X$ is connected and strongly 1-acyclic over $Z_{p}$ and that some neighborhood of $X$ in $M^{3}$ contains no $Z_{p}$-homology 3-cells which fail to be simply connected. Then $X$ is strongly acyclic over $Z_{p}$ if and only if $X$ has property 2-UV.

Although the following result and its corollary will not be needed 
for the later proofs, we think they have interest for their own sake. They may be regarded as extensions of the theorem of Case and Chamberlin [10, Theorem 1] to the effect that a 1-dimensional continuum $X$ is treelike if and only if $X$ admits no essential mapping into a finite, connected graph. It is not hard to show that a compact set $X \subset E^{n}$ has property $\mathrm{UV}^{\infty}$ if and only if $X$ admits an essential map into no finite polyhedron of dimension $\leqq(n-1)$.

Proposition. Let $X$ be a compact set which embeds in $E^{3}$. Then $X$ has property 1-UV if and only if $X$ admits no essential mapping into a wedge $W$ of two 1-spheres.

Proof. We may assume that $X$ is a subset of $E^{3}$. Any mapping of $X$ into $W$ extends to an open set in $E^{3}$ containing $X$, and hence cannot be essential if $X$ has property 1-UV (recall that $W$ is aspherical, i.e., $\pi_{i}(W)=0$ for $i \geqq 2$, and hence a mapping of a finite polyhedron into $W$ is inessential if and only if it induces the trivial homomorphism on fundamental groups).

Now suppose that $X$ admits no essential mapping into $W$. Then no component of $X$ admits such a mapping, and it suffices to establish our result with the added hypothesis that $X$ is a continuum in $E^{3}$. Since $S^{1}$ is a retract of $W$, there is no essential mapping of $X$ into $S^{1}$. We shall use this last fact to show that $X$ is strongly 1 -acyclic over $Z$. By Lemma 1, part (ii), we have only to show that each polyhedral simple closed curve $J$ in $E^{3}-X$, when considered as a 1 -cycle over $Z$, is homologous to zero in $E^{3}-X$.

Note that the "abelianizer" homomorphism of $\pi_{1}\left(E^{3}-J\right)$ onto $Z$ sends each loop in $E^{3}-J$ to its integral linking number with $J$. Using the asphericity of $S^{1}$, we can construct a mapping $R: E^{3}-J \rightarrow S^{1}$ which induces this abelianizer homomorphism on fundamental groups. (In fact, we could use the technique of [13, Theorem 5] to construct an appropriate retraction.) The composition of the inclusion $X \rightarrow E^{3}$ $-J$ with the mapping $R$ is by hypothesis an inessential mapping of $X$ into $S^{1}$. Hence there is a neighborhood $N$ of $X$ in $E^{3}-J$ such that each simple closed curve in $N$ has integral linking number zero with $J$. By repeating the type of argument required for the "only if" claim of Lemma 1, part (ii), we find that $J$ is homologous to zero in $E^{3}-X$.

It follows from the above that $X$ is strongly 1-acyclic over $Z$. Thus our Corollary 2.1 applies. Let $H_{1}, H_{2}, \cdots$ be the 3 -manifolds given by that corollary's conclusion, with $X=\bigcap_{i=1}^{\infty} H_{i}$. Each $Q^{3}$ mentioned in Corollary 2.1 is bounded by 2 -spheres, and hence in this case is simply connected. Fix a value of $i$. Then $H_{i}$ is a regular 
neighborhood of a finite wedge $A_{i}$ of 1 -spheres and 2-spheres (the number of $j$-spheres in $A_{i}$ may be zero, $j=1,2$ ). But given such a wedge $A_{i}$, some mapping of $A_{i}$ into $W$ induces a monomorphism on fundamental groups (first retract the 2 -spheres to the wedge point, then apply problem 2 of $[21$, p. 112] to complete the construction). Thus, some mapping $R_{i}: H_{i} \rightarrow W$ induces a monomorphism on fundamental groups.

Since $R_{i} \mid X$ is an inessential map of $X$ into $W$, there is a neighborhood $N$ of $X$ in $H_{i}$ such that $R_{i} \mid X$ extends to an inessential map $F_{i}$ of $N$ into $W$. If now $N$ is further restricted, say to $N_{0}\left(X \subset N_{0} \subset N\right)$, we may then assume that $F_{i} \mid N_{0}$ and $R_{i} \mid N_{0}$ are homotopic mappings into $W$. Hence $R_{i} \mid N_{0}$ is inessential. By our "monomorphism" property of $R_{i}$, the inclusion $N_{0} \rightarrow H_{i}$ induces the trivial homomorphism on fundamental groups. The result follows.

REMARK. Similar methods show that a compact set $X \subset E^{3}$ is strongly acyclic over $Z$ if and only if $X$ admits no essential mapping into $S^{n}(n=0,1,2)$. Finally, there is a combined formulation of these results which seems especially appealing.

Corollary. Let $X$ be a compact set which embeds in $E^{3}$. Then $X$ has property $\mathrm{UV}^{\infty}$ if and only if $X$ admits no essential mapping into the following finite 2-complex: the disjoint union of $S^{2}$ and a wedge of two 1-spheres.

3. Detecting 1-UV elements in acyclic decompositions. The next theorem and its corollaries are among our most useful results. In its proof and later, let us agree to call a set $S \subset M^{k}$ saturated (with respect to the mapping $P: M^{k} \rightarrow Y$ ), if $P^{-1} P(S)=S$. The reader should note that the "UV" type requirement for $P^{-1}\left(y_{0}\right)$ in Theorem 3 , is met whenever $Y$ is locally simply connected at $y_{0}$. This means that each neighborhood $N$ of $y_{0}$ in $Y$ contains a neighborhood $W$ of $y_{0}$ such that each loop in $W$ is contractible in $N$.

Let $\alpha$ be an ordinal, and let $p$ denote 0 or a prime. A compact, connected set $X \subset M^{k}$ has an $(\alpha, p)$-trivial $\pi_{n}$-shape $(n \geqq 1)$ if for each open, connected set $U \subset M^{k}$ such that $X \subset U$, there is an open set $V, X \subset V \subset U$, such that each singular $n$-sphere in $V$ represents an element belonging to the term $F_{\alpha}$ of the $p$-central series of the group $F=\pi_{n}(U)$. As expected, this is a topological property of $X$ with respect to embeddings in manifolds.

The "local connection" terminology of Corollary 3.1 is due to George Kozlowski [16]. A special case of Corollary 3.1 has been proven by Alden Wright in [35].

THEOREM 3. Let $M^{k}$ be a k-manifold, possibly with boundary, and 
let $P$ be a compact, monotone mapping of $M^{k}$ onto a nondegenerate Hausdorff space $Y$. Suppose that for each $y \in Y, P^{-1}(y)$ is strongly 1-acyclic over $Z_{p}(p=0$ or a prime). Suppose that the following property holds for some $y_{0} \in Y:$ For each open set $U \subset M^{k}$ such that $P^{-1}\left(y_{0}\right) \subset U$, there is an open set $V, P^{-1}\left(y_{0}\right) \subset V \subset U$, such that each loop in $V$ projects under $P$ to a contractible loop in $P(U)$. Then $P^{-1}\left(y_{0}\right)$ has an $(\omega, p)$-trivial $\pi_{1}$-shape. Hence, if $P^{-1}\left(y_{0}\right)$ embeds in an orientable, nonclosed 3-manifold $\left(\right.$ e.g., $\left.E^{3}\right)$ containing no $Z_{p}$-homology 3-cells which fail to be simply connected, then $P^{-1}\left(y_{0}\right)$ has property 1-UV.

Proof. Note that $X=P^{-1}\left(y_{0}\right)$ is a proper, compact connected subset of $M^{k}$. Let an open, connected set $U \subset M^{k}$ be given, $X \subset U$. Since $P$ is a closed mapping, $P(U)$ contains a neighborhood of $y_{0} \in Y$, and so there are saturated, open, connected sets $V, V_{0}$, with

$$
X \subset V \subset V_{0} \subset P^{-1} P\left(\bar{V}_{0}\right) \subset U,
$$

and such that each loop in $V$ projects under $P$ to a loop which is contractible to a point in $P\left(V_{0}\right)$.

Let $G$ be the monotone decomposition of $U$ consisting of the sets $P^{-1} P(z)$ for all $z \in \bar{V}_{0}$, together with the individual points of $U$ $-P^{-1} P\left(\bar{V}_{0}\right) . G$ is upper semicontinuous because $P^{-1} P\left(\bar{V}_{0}\right)$ is a saturated, closed set in $M^{k}$ and because

$$
\left\{P^{-1}(y): y \in Y\right\}
$$

is an upper semicontinuous decomposition of $M^{k}$. Further, each loop in $V$ projects to a contractible loop in the decomposition space $U / G$. Hence, applying Corollary 1.1 (with $n=1$ ) to the projection mapping $U \rightarrow U / G$, we find that each loop in $V$ represents an element belonging to the term $F_{\omega}$ of the $p$-central series of the group $F=\pi_{1}(U)$. The result follows.

For our last conclusion, suppose that $X^{*} \subset M^{3}$ is a homeomorph of $X$, where $M^{3}$ is an orientable, nonclosed piecewise-linear 3-manifold which contains no $Z_{p}$-homology 3 -cells which fail to be simply connected. We may assume that $X^{*} \subset$ Int $M^{3}$, and it suffices to show that $X^{*}$ has property $1-\mathrm{UV}$.

If $U$ is a given neighborhood of $X^{*}$ in $M^{3}$, some polyhedral neighborhood $H^{3}$ of $X^{*}$ in $U$ has the structure described in Corollary 2.1. Moreover, we may demand in this case that the 3 -manifold $Q^{3}$ in that corollary's conclusion be simply connected (see Remark 1 following Corollary 2.1.) In particular, $\pi_{1}\left(H^{3}\right)$ is a free group. Let $V$ be a neighborhood of $X^{*}$ in $H^{3}$ such that each loop in $V$ represents an element belonging to the term $F_{\omega}$ of the $p$-central series of the free group $F=\pi_{1}\left(H^{3}\right)$. (Such exists by the first part of our proof.) 
But $F_{\omega}=1$ by Theorem 6.3 of [32]. This completes our proof.

Corollary 3.1. Let $M^{3}$ be a piecewise-linear 3-manifold, possibly with boundary, and let $P$ be a compact mapping of $M^{3}$ onto a nondegenerate Hausdorff space $Y$. Suppose that for each $y \in Y, P^{-1}(y)$ is strongly 1-acyclic over $Z_{p}$ and has an orientable neighborhood in $M^{3}$ (if $p>2$ ). Suppose that $P$ is a local connection in dimension 1. Then, for all but a discrete set $D$ of points $y \in Y, P^{-1}(y)$ has property 1-UV. In fact, for each compact 3-manifold $N^{3}$ (possibly with $N^{3}-M^{3} \neq \varnothing$ ), there exist only a finite number of $y \in D$ for which $P^{-1}(y) \subset N^{3}$.

Proof. The requirement that $P$ be a local connection in dimension 1 yields two facts: $P$ is monotone, and the hypothesis of Theorem 3 holds for each $y_{0} \in Y$. We need only the additional information that a compact 3-manifold $N^{3}$ contains but a boundedly-finite number of disjoint $Z_{p}$-homology 3 -cells which fail to be simply connected. For more details on this last matter, see [35]. An application of Theorem 3 then completes the proof.

Recall that a compact decomposition of $M^{3}$ is one whose elements consist of the components of some compact set $K \subset M^{3}$, plus the individual points of $M^{3}-K$. We adopt the convention that a compact decomposition of $M^{3}$ must yield a nondegenerate decomposition space. Thus, for each compact, proper subset of $M^{3}$, we may speak of the compact decomposition of $M^{3}$ associated with $K$. If $K$ is also connected, then its associated decomposition space is usually called $M^{3}$ modulo $K$.

If $G$ is an upper semicontinuous decomposition of $M^{3}$, let $H_{G}$ denote the union of the nondegenerate elements of $G$, and let $P_{G}$ denote the projection mapping of $M^{3}$ onto the decomposition space $M^{3} / G$. In particular, a compact decomposition $G$ of $M^{3}$ is the compact decomposition of $M^{3}$ associated with $\bar{H}_{G}$, and $P_{G}\left(\bar{H}_{G}\right)$ is compact and 0-dimensional. If $F$ and $G$ are compact decompositions of $M^{3}$, then, following [5], we say that $F$ is equivalent to $G$ if some homeomorphism $h$ of $M^{3} / F$ onto $M^{3} / G$ carries $P_{F}\left(\bar{H}_{F}\right)$ onto $P_{G}\left(\bar{H}_{G}\right)$. We call $h$ an equivalence from $F$ to $G$. A compact decomposition $G$ of $M^{3}$ is strongly acyclic over $Z_{p}$ if each component of $\bar{H}_{G}$ is strongly acyclic over $Z_{p}$.

We now draw several conclusions about equivalent compact decompositions. For simplicity of statement, we restrict ourselves to the consideration of $E^{3}$.

Corollary 3.2. Let $F$ and $G$ be compact decompositions of $E^{3}$. Suppose that $F$ is strongly acyclic over $Z_{p}$, and that $h$ is an equivalence 
from $F$ to $G$. Then $G$ is strongly acyclic over $Z_{p}$. Further, if $h\left(P_{F}(f)\right)$ $=P_{G}(g)$ for some $f \in F$ and $g \in G$, then $f$ has property $U^{\infty}$ if and only if $g$ has property $\mathrm{UV}^{\infty}$.

Proof. The strong acyclicity of $G$ follows from Lemma 1, parts (ii) and (iii). Now suppose that $f$ has property $\mathrm{UV}^{\infty}$. We claim that $P_{G}$ is a "local connection in dimension 1, at $g$ " in the sense that the last part of the hypothesis of Theorem 3 holds (with $g=P_{G}^{-1}\left(y_{0}\right)$ ). Theorem 3 then will reveal that $g$ has property 1-UV, and hence property $\mathrm{UV}^{\infty}$.

To prove our claim, let a connected, open set $U \subset E^{3}, g \subset U$, be given. Since $G$ is strongly acyclic over $Z_{p}$, Corollary 2.1 provides a polyhedral cube-with-handles $V^{3} \subset U, g \subset \operatorname{Int} V^{3}$, such that $\bar{H}_{G} \cap \partial V^{3}$ $=\varnothing$. Further, by the UV ${ }^{\infty}$ property of $f$, we may suppose further that $P_{F}^{-1} h^{-1} P_{G}\left(V^{3}\right)$ is contractible to a point in $P_{F}^{-1} h^{-1} P_{G}(U)$. Now a given loop in $V^{3}$ is freely homotopic in $V^{3}$ to a loop $t_{1}$ in $\partial V^{3}$. But the loop $t_{0}=P_{F}^{-1} h^{-1} P_{G^{i}}{ }^{1}$ contracts in $P_{F}^{-1} h^{-1} P_{G}(U)$. Thus $h P_{F} t_{0}=P_{G} t_{1}$ contracts in $P_{G}(U)$. This establishes our claim. Previous remarks and symmetry complete the proof.

REMARK. It follows from Corollary 3.2 that if the complement of a compact set $X \subset E^{3}$ is homeomorphic to the complement of some compact set with property $\mathrm{UV}^{\infty}$, then $X$ also has property $\mathrm{UV}^{\infty}$. Further, by our next corollary, $E^{3}-X$ is homeomorphic to the complement in $E^{3}$ of a treelike continuum.

Corollary 3.3. Let the compact decomposition $F$ of $E^{3}$ be strongly acyclic over $Z_{p}$. Then there is a compact decomposition $G$ of $E^{3}$, with $\bar{H}_{G}$ one-dimensional, and an equivalence $h$ from $F$ to $G$. Further, $G$ is strongly acyclic over $Z_{p}$, and if $h\left(P_{F}(f)\right)=P_{G}(g)$, for some $f \in F$ with property $\mathrm{UV}^{\infty}$ and for some $g \in G$, then $g$ also has property $\mathrm{UV}^{\infty}$ (and hence is treelike).

Proof. This is immediate from our Corollary 2.1, [5, Theorem 8], and the previous corollary. For the parenthetical remark, see [10, Theorem 1], or see [33, Theorem 2], and recall that a compact set in $E^{n}$ with property $U^{\infty}{ }^{\infty}$, is cellular in $E^{n+1}$ (cf. the second paragraph of the introduction to [24] and [24, Corollary to Theorem 8]).

Corollary 3.4. Let $G$ be a compact decomposition of $E^{3}$. Suppose that $\left(E^{3} / G\right) \times E^{k}$ is topologically $E^{3+k}$ for some integer $k$. Then each component of $\bar{H}_{G}$ has property $\mathrm{UV}^{\infty}$.

Proof. Note that $E^{3} / G$, as a retract of $E^{3+k}$, is locally contractible. Hence, the result will follow from Theorem 3 , once it is shown that $\bar{H}_{G}$ is strongly 1-acyclic over $Z_{2}$ and has property 2-UV. 
Let $G^{*}$ be the upper semicontinuous decomposition of $E^{3+k}$ whose nondegenerate elements are precisely the nondegenerate sets of the form $g \times\{t\}$, where $g \in G$ and $t \in E^{k}$. Then there is a compact mapping $P$ of $E^{3+k}$ onto $E^{3+k}$ whose induced decomposition into pointinverse sets is precisely $G^{*}$.

Let $\hat{P}: S^{3+k} \rightarrow S^{3+k}$ be the obvious extension of $P$ to the one-point compactifications $S^{3+k}=E^{3+k} \cup\left\{p_{\infty}\right\}$. Let $S=\left(\bar{H}_{a} \times E^{k}\right) \cup p_{\infty}$, and note that $\hat{P} \mid S^{3+k}-S$ is a homeomorphism onto $S^{3+k}-\hat{P}(S)$. But $S^{3+k}-S$ is topologically $\left(E^{3}-\bar{H}_{G}\right) \times E^{k}$, and hence $E^{3}-\bar{H}_{G}$ has the homotopy type of $S^{3+k}$ minus the compact, $k$-dimensional set $\hat{P}(S)$. Since $\hat{P}(S)$ is strongly $(k+1)$-acyclic over $Z_{2}$ and does not separate $S^{3+k}$, it follows that $H_{1}\left(E^{3}-\bar{H}_{G} ; Z_{2}\right)=0$, and that $E^{3}-\bar{H}_{G}$ is connected. According to Lemma $1, \bar{H}_{G}$ is strongly 1 -acyclic over $Z_{2}$ and has property 2-UV. This completes the proof.

Our next corollary is known in the case that $k=3$ and $P_{G}\left(\bar{H}_{G}\right)$ is compact and 0 -dimensional. Independent proofs of this case were given by the author in [26, Addendum 2 to Theorem 5], and by H. W. Lambert in [20].

COROLlaRY 3.5. Let $G$ be an upper semicontinuous decomposition of $E^{k}$ into compact, connected sets such that the decomposition space $E^{k} / G$ is an open k-manifold. Suppose that $P_{G}\left(H_{G}\right)$ is 0-dimensional. If $k \neq 4$, then $E^{k} / G$ is topologically $E^{k}$. If an element $g_{0} \in G$ admits an embedding in $E^{3}$, then $g_{0}$ has property $\mathrm{UV}^{\infty}$. In particular, if each $g \in G$ embeds in $E^{3}$ and $k \neq 4$ (for example, suppose $k=3$ ), then $G$ is a cellular decomposition of $E^{k}$.

PRoof. If $k \leqq 2$, then the hypotheses of the first sentence of our corollary imply that $G$ is cellular and that $Y^{k}=E^{k} / G$ is topologically $E^{k}$. Hence, assume $k \geqq 3$.

By Lemma 5 below, each $g \in G$ is strongly acyclic over $Z$, and hence $H_{i}\left(Y^{k} ; Z\right)=0$ for $i>0$. Further, $P_{G}: E^{k} \rightarrow Y^{k}$ is monotone, so that $Y^{k}$ is simply connected (see [16], e.g.). Thus, $Y^{k}$ is contractible. Again using the fact that $P_{G}$ is monotone (and the resulting " $\pi_{1^{-}}$ surjection" property), it follows that $Y^{k}$ is $1-\mathrm{LC}$ at infinity, in the sense of [28]. Hence, for $k>4, Y^{k}$ is topologically $E^{k}$ by [28, Theorem $1.1]$. For $k=3, Y^{3}$ is topologically $E^{3}$ because of [6, Theorem 3$]$ and the fact that $G$ is a cellular decomposition, as will be indicated.

If some $g_{0} \in G$ embeds in $E^{3}$, then $g_{0}$ has property $\mathrm{UV}^{\infty}$ by our Theorem 3 and [19]. If each $g \in G$ embeds in $E^{3}$, then each $g \in G$ has property $U V^{\infty}$ and if $k \neq 4, G$ is cellular by, e.g. [ 1 , Theorems 5.5 and 5.6]. (See also [27], [22], [25], [15], and [19].)

We now present a sequence of lemmas leading to a proof of the 
acyclicity claim needed above. The first lemma is an exercise in excision, and its proof is omitted.

Lemma 2. Let $Z \subset W \subset M^{k}$, where $Z$ is compact, and $W$ is an open, connected subset of the open $k$-manifold $M^{k}$. If a (finite, singular) integral $i$-cycle in $W$ is homologous to zero in $M^{k}$ then it is homologous in $W$ to an $i$-cycle in $W-Z$.

Suppose $\mathfrak{U}$ is a collection of subsets of the topological space $Y$, and $B C Y$. The star of $B$ with respect to $\mathfrak{u}$, or $\operatorname{st}(B, \mathcal{u})$, is the union of all elements of $\mathcal{u}$ which intersect $B$. The union of all the sets in $u$ is denoted by $\mathcal{u}^{*}$. If $\mathcal{U}$ and $\mathcal{V}$ are collections of open sets in $Y$, then (as in [6]) $\mathcal{U}$ star $n$-homotopy refines $\mathcal{U}$ if for each $V \in \mathcal{V}$ there exists $U \in \mathcal{U}$ such that $\operatorname{st}(V, \mathcal{V}) \subset U$, and for $0 \leqq k \leqq n$, each singular $k$ sphere in $\operatorname{st}(V, V)$ is contractible in $U$.

Convention. For Lemmas 3 and 4, assume the following notation. $X$ is an $\mathrm{LC}^{n-1}(n \geqq 1)$, locally compact metric space. $G$ is an upper semicontinuous decomposition of $X$ into compact, connected sets. (The decomposition space $Y=X / G$ is necessarily metrizable.)

Lemma 3. For each collection $u$ of open sets in $Y$, there is a collection $\vartheta$ of open sets in $Y$ such that $P_{G}^{-1}(\mathcal{V})$ star $(n-1)$-homotopy refines $P_{G}^{-1}(\mathcal{u})$, and $u^{*}-\vartheta^{*} \subset P_{G}\left(H_{G}\right)$.

Proof. The proof is a variation of that of [6, Lemma 3.1]. Suppose $\mathcal{U}$ is given. Let $\mathcal{U}^{*}=B \subset Y$ and $P_{\theta}^{-1}(B)=A \subset X$. Let $K_{1}, K_{2}, \cdots$ be a locally finite (with respect to $A$ ) collection of compact sets in $A$ whose interiors cover $A$. There is an $\epsilon_{i}>0$ such that if a set $S \subset X$ has diameter less than $\epsilon_{i}$ and if $S \cap K_{i} \neq \varnothing$, then $S \subset R$, an open set in $A$ such that for $0 \leqq k \leqq n-1$, each singular $k$-sphere in $R$ is contractible to a point in an element of $P_{G}^{-1}(\mathcal{U})$. Let $M_{i}$ be the union of all $g \in G$ such that $g$ intersects $K_{i}$ and $g$ has diameter at least $\epsilon_{i}$. By [34, Corollory 2.61], each set $P_{G}^{-1} P_{G}\left(K_{i}\right)$ is compact, and so $M_{1}$, $M_{2}, \cdots$ is a locally finite collection of compact sets in $A$. Thus, $M=\bigcup_{i=1}^{\infty} M_{\imath}$ is closed in $A$.

Define $C=P_{G}(M)$, a relatively closed set in $B$. Clearly, if $y \in B-C$, then there is a saturated open set $W_{\nu} \subset A-M$ such that $P_{G}^{-1}(y) \subset W_{\nu}$, and such that for $0 \leqq k \leqq n-1$, each singular $k$-sphere in $W_{\nu}$ is contractible to a point in some element of $P_{G}^{-1}(\mathcal{U})$. Thus,

$$
\mathscr{W}=\left\{P_{G}\left(W_{y}\right): y \in B-C\right\}
$$

is an open covering of $B-C$. We take $v$ to be any open star refinement of $W$ covering $B-C$. The proof is complete.

Lemma 4. For each open covering $\mathfrak{u}$ of $Y$, there is a closed set 
$C \subset P_{G}\left(H_{G}\right)$ ( $C$ depends only upon $\mathcal{u}$ and $n$ ) such that the following holds: Suppose $g$ is a map from a finite simplicial complex $K$ of $d i$ mension $n$ or less, into $Y-C$, and $f$ is a map from a subcomplex $L$ of $K$ into $X-P_{G}^{-1}(C)$, such that $g \mid L=P_{G} f$. Then $f$ extends to a map $F$ sending $K$ into $X$ such that for each $z \in K$, there exists an element of $u$ containing both $g(z)$ and $P_{G} F(z)$.

Proof. The proof is similar to that of [6, Lemma 3.2]. Suppose that $\mathfrak{u}$ and $n$ are specified. Let $\mathfrak{u}_{n}$ be an open covering of $Y$ which star refines $\mathfrak{u}$. By Lemma 3 , there are collections $\mathfrak{u}_{n-1}, \mathfrak{u}_{n-2}, \cdots, \mathfrak{u}_{0}$ of open sets in $Y$ such that (for $0 \leqq i \leqq n-1): P_{G}^{-1}\left(\mathcal{U}_{i}\right)$ star $(n-1)$ homotopy refines $P_{G}^{-1}\left(\mathcal{u}_{i+1}\right)$, and

$$
u_{i+1}^{*}-u_{i}^{*} \subset P_{G}\left(H_{G}\right) \text {. }
$$

Define $C=Y-\mathcal{u}_{0}^{*}$. Clearly, $C \subset P_{G}\left(H_{G}\right)$ is closed.

Now suppose that maps $f$ and $g$ are given as described above. Assume that $\operatorname{dim} K=n$. We construct an extension $F$ of $f$ to $K$, essentially as in [6, Lemma 3.2]. (We begin with the second paragraph of their proof, noting that their $\mathcal{u}_{i}$ 's cover $X$ rather than $Y$.)

Briefly, find a subdivision $T$ of $K$ so that for each $\sigma \in T, g(\sigma)$ lies in some element of $\mathcal{u}_{0}$. We construct inductively a sequence of maps

$$
F_{i}: T^{i} \rightarrow P_{G}^{-1}\left(\mathcal{U}_{i}^{*}\right), \quad 0 \leqq i \leqq n,
$$

$\left(T^{i}=i\right.$-skeleton of $T$ ) such that

(1) if $i>0, F_{i}$ extends $F_{i-1}$,

(2) $F_{i}$ agrees with $f$ on $|L| \cap\left|T^{i}\right|$, and

(3) if $\sigma^{i} \in T$, then $F_{i}\left(\sigma^{i}\right) \subset$ some element of $P_{G}^{-1}\left(\mathcal{U}_{i}\right)$. We leave to the reader the minor changes necessary in the construction of [6].

In place of the last paragraph of their proof, we have only to note that for each $z \in K, g(z)$ and $P_{G} F(z)$ lie in intersecting elements of $\mathcal{u}_{n}$, and hence some element of $\mathcal{u}$ contains them both. In fact, if $\sigma$ is a simplex of $T$ containing $z$, then $P_{G} F(\sigma)$ lies in some element of $\mathcal{u}_{n}$, and $g(\sigma)$ lies in some element of $\mathcal{u}_{n}$. These two elements meet in the point $g(v)=P_{G} F(v)$, where $v$ is a vertex of $\sigma$.

The reader may wish to compare our next result with those of [29]. The homology and cohomology groups below are understood to have $Z$ coefficients. In [19], Lacher has shown that a compact, connected set $g \subset M^{k}$ is strongly acyclic over $Z$ if and only if it is cohomologically trivial over $Z$. More precisely, properties $(k-1)$ $\mathrm{uv}(Z)$ and $k$-uv $(Z)$ together imply $\check{H}^{k}=0$. Conversely, if $\check{H}^{k}=\check{H}^{k+1}$ $=0$ for $g$, then $g$ has property $k-\mathrm{uv}(Z)$.

LEMma 5. Let $G$ be an upper semicontinuous decomposition of an 
open $k$-manifold $M^{k}$ into compact, connected sets such that the decomposition space $Y^{k}=M^{k} / G$ is also an open $k$-manifold, $k \geqq 3$. Suppose that $P_{G}\left(H_{G}\right)$ is 0-dimensional. Suppose also that the following holds (as it does, e.g., if $\left.M^{k}=E^{k}\right)$ : For each $g \in G$, there is an open set $U_{g}$, $g \subset U_{o} \subset M^{k}$, such that under inclusion,

$$
H_{i}\left(U_{g}\right) \rightarrow H_{i}\left(M^{k}\right)
$$

is zero for $i=1, \cdots, n=[k / 2]$. Then each $g \in G$ is strongly acyclic over $Z$.

Proof. Note that since $n \geqq 1$, each $U_{\theta}$ is orientable. We will show that each $g \in G$ has property $i$-uv $(Z), i=1, \cdots, n$, and hence is cohomologically trivial (over $Z$ ) in dimensions $1, \cdots, n$. By the proof given for [17, Theorem 4] (the statement in [17] is incorrect), it will then follow that $\check{H}^{*}(g)=0$, for each $g \in G$, and hence that each $g \in G$ has $\operatorname{uv}^{\infty}(Z)$.

Our goal, then, is to prove that if $B^{k}$ is an open $k$-cell in $Y^{k}$ such that $W=P_{G}^{-1}\left(B^{k}\right)$ has compact closure and is contained in some $U_{g}$, then $H_{i}(W)=0$ for $1 \leqq i \leqq n$. Note that $W$ is connected, because $G$ is monotone.

By Lemma 4, there is a closed (in $B^{k}$ ) 0-dimensional set $C_{1} \subset P_{G}\left(H_{G}\right)$ corresponding to the one-element covering of $B^{k}$, and to the integer $(k-1)$. Let $v$ be an open covering of $B^{k}-C_{1}$ with this property: If $f_{1}, f_{2}$ are mappings of any space $Z$ into $B^{k}-C_{1}$ and if for each $z \in Z$ there exists some element of $V$ containing both $f_{1}(z)$ and $f_{2}(z)$, then $f_{1}$ and $f_{2}$ are homotopic as mappings of $Z$ into $B^{k}-C_{1}$. Finally, apply Lemma 4 (with $Y=B^{k}-C_{1}$ ) to obtain a closed (in $\left.B^{k}-C_{1}\right)$ set $C_{2}^{\prime} \subset P_{G}\left(H_{G}\right)$, corresponding to $V$ and to the integer $k$. Put $C_{2}=C_{1} \cup C_{2}^{\prime}$, a relatively closed set in $B^{k}$. Let $C_{j}^{*}$ denote $P_{G}^{-1}\left(C_{j}\right)$, $j=1,2$.

Consider portions of the two exact homology sequences of pairs below, and the vertical homomorphisms between them induced by $P_{G}$ :

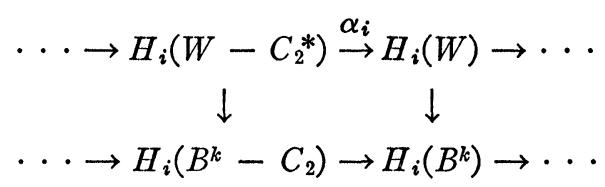

An easy argument using [34, Corollary 2.61] and our Lemma 2, reveals that $\alpha_{i}$ is an epimorphism for $i \leqq n$. We claim also that $\alpha_{i}$ is zero for $i \leqq n$. The proof of this assertion will complete our argument. 
Suppose, then, that $x \in H_{i}\left(W-C_{2}^{*}\right)$, where $i \leqq n$ is fixed. Construct an oriented, homogeneously $i$-dimensional, finite polyhedron $Q$ whose combinatorial boundary (over $Z$ ) is zero, together with a map $h: Q \rightarrow W-C_{2}^{*}$ whose induced homomorphism $h_{*}$ takes the orientation class $c_{Q}$ of $Q$, to $x$. Since $H_{i}\left(B^{k}-C_{2}\right)=0$ (because $i \leqq k-2$ ), there is a finite, $k$-dimensional polyhedron $K \subset B^{k}-C_{2}$ such that if $\pi=P_{G} h$, then $\pi(Q) \subset K$ and $\pi_{*}\left(c_{Q}\right)=0 \in H_{i}(K)$. Consider the consistent diagram

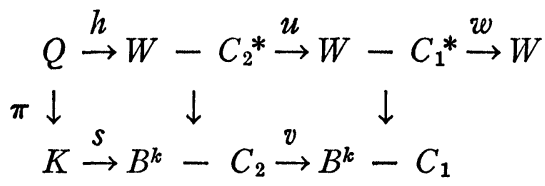

in which the horizontal maps, other than $h$, are inclusions, and the vertical maps, other than $\pi$, are restrictions of $P_{G}$.

By our choice of $C_{2}$ and $\mathcal{V}$, there is a map $S: K \rightarrow W-C_{1}^{*}$ such that $P_{G} S$ and $v s$ are homotopic maps of $K$ into $B^{k}-C_{1}$. Hence, $P_{G} u h$ and $P_{G} S \pi$ are homotopic maps of $Q$ into $B^{k}-C_{1}$. By our choice of $C_{1}$, wuh and $w S \pi$ are homotopic maps of $Q$ into $W$. Thus,

$$
\left(\alpha_{i}\right)_{*}(x)=w_{*} u_{*} h_{*}\left(c_{Q}\right)=w_{*} S_{*} \pi_{*}\left(c_{Q}\right)=0 \in H_{i}(W) .
$$

This is the desired result.

EXAMPLE. There is a continuous, onto mapping $P: S^{4} \rightarrow S^{4}$ such that each $P^{-1}(y)$ is strongly acyclic over $Z$, yet some point-inverses fail to have property 1-UV. For as Mazur shows in [23], $S^{4}$ can be expressed as the "double" of a certain compact, contractible 4-manifold whose boundary $M^{3}$ has the homology (over $Z$ ) of $S^{3}$, but $M^{3}$ fails to be simply connected. There is a piecewise-linear homeomorphism $h$ of $M^{3} \times[-1,1]$ onto a neighborhood $N$ of $M^{3}$ in $S^{4}$. Let $K$ be $M^{3}$ minus the interior of a 3 -simplex, so that $\pi_{1}(K) \approx \pi_{1}\left(M^{3}\right)$. Then the nondegenerate point-inverses of $P$ are the sets $h(K \times\{t\}), t \in(-1,1)$, plus the closures of the two components of $S^{4}-N$. Similar examples of maps $S^{n} \rightarrow S^{n}$ are possible for each $n \geqq 4$.

4. A monomorphism theorem and some applications. If $f: X \rightarrow Y$ is a map, let $S_{f} \subset X$ be the singular set of $f$. That is,

$$
S_{f}=\left\{x \in X: f^{-1} f(x) \neq x\right\} .
$$

It seems of interest to note here that $P_{*}$ in Theorem 4 below may fail to be an isomorphism. In fact, R. H. Bing has announced in [7] that $E^{3}$ modulo a solenoid is not simply connected. 
Theorem 4. Let $M^{3}$ be a piecewise-linear 3-manifold, possibly with boundary, and let $P$ be a compact, monotone mapping of $M^{3}$ onto a Hausdorff space $Y$. Suppose that there is a compact set $A \subset M^{3}$ such that $\bar{S}_{p} \subset A$ and such that each component of $A$ is strongly 1-acyclic over $Z_{p}$, where $p=0$ or a prime. If some compact, polyhedral 3-manifold $H$ has $\pi_{1}(H)$ free and if $A \subset H \subset M^{3}$, then the kernel of the homomorphism

$$
P_{*}: \pi_{1}\left(M^{3}, x_{0}\right) \rightarrow \pi_{1}\left(Y, P\left(x_{0}\right)\right)
$$

is trivial.

Proof. There is a compact, monotone mapping $P_{0}$ of $M^{3}$ onto a Hausdorff space $Y_{0}$ such that the nondegenerate point-inverses of $P_{0}$ are precisely the nondegenerate components of $A$, and such that $P_{0} P^{-1}$ is a well-defined mapping of $Y$ onto $Y_{0}$. Further, if $P_{0}$ induces a monomorphism on fundamental groups, so also does $P$. Hence, we assume without loss of generality that the nondegenerate pointinverses of $P$ are precisely the nondegenerate components of $A$. (We shall later need the fact that $P(A)$ is compact and 0-dimensional.)

Let $L=\partial \Delta^{2}$. Suppose that $g: \Delta^{2} \rightarrow Y$ and $f: L \rightarrow M^{3}$ are maps with $g \mid L=P f$. We wish to show that $f$ is homotopic in $M^{3}$ to a constant map. Since $H \cup P^{-1} g\left(\Delta^{2}\right)$ is compact, we may assume, by the existence of regular neighborhoods, that $M^{3}$ is compact. By attaching a collar to $\partial M^{3}$ and redefining $Y$, if necessary, we may also suppose that $A \subset$ Int $H$ and $P^{-1} g\left(\Delta^{2}\right) \subset$ Int $M^{3}$.

Let $T$ be a triangulation of $M^{3}$ in which $H$ appears as a full subcomplex. There is a finite, polyhedral graph $\Gamma$ in $M^{3}-H$ such that the complement of a close, open regular neighborhood of $\Gamma$ is a regular neighborhood of $H \cup T^{1}$. For example, take $\Gamma$ to be that subcomplex of the first-derived $T^{\prime}$ which is maximal with respect to not intersecting $H \cup T^{1}$. In particular, $\pi_{1}\left(M^{3}-\Gamma\right)$ is a free group. By performing a homotopy, if necessary, we may assume that $f(L)$ misses $\Gamma$, and we may further adjust $g$ so that $g^{-1} P(\Gamma)$ is a finite set.

Let $\alpha_{1}, \cdots, \alpha_{k}$ be a finite, disjoint collection (possibly empty) of polyhedral arcs in $\Delta^{2}-g^{-1} P(A)$ such that each $\alpha_{i}$ intersects each of $\partial \Delta^{2}, g^{-1} P(\Gamma)$ precisely in one of its endpoints, and such that no arc in $\Delta^{2}-g^{-1} P(A)$ joins a point of $g^{-1} P(\Gamma)-\bigcup_{i=1}^{k} \alpha_{i}$ to $\partial \Delta^{2}$. After removing close, disjoint regular neighborhoods of the $\alpha_{i}$ 's in $\Delta^{2}-g^{-1} P(A)$ and redefining $g, f$, etc., we can assume that no component of $\Delta^{2}-g^{-1} P(A)$ intersects both $g^{-1} P(\Gamma)$ and $\partial \Delta^{2}$.

We now claim that $P f$ is homotopic to a constant map in $Y-P(\Gamma)$. If this can be shown, our proof will be complete. For an application of Corollary 1.1 (with $n=1$ ) to the 3-manifold $M^{3}-\Gamma$ then will reveal 
that the element of $\pi_{1}\left(M^{3}-\Gamma\right)$ represented by $f$ belongs to the $\omega$ th term of the $p$-central series of that free group, and hence represents its identity element (see [32, Theorem 6.3]).

If $g^{-1} P(\Gamma)=\varnothing$, then the claim of the previous paragraph is obvious. Suppose $U$ is a component of $\Delta^{2}-g^{-1} P(A)$ with $U \cap g^{-1} P(\Gamma)$ $\neq \varnothing$ (so that $U \subset \operatorname{Int} \Delta^{2}$ ). Let $D \subset U$ be a polyhedral disk whose interior contains $U \cap g^{-1} P(\Gamma)$. To establish our claim, it suffices to show that for each such $U$ and $D$, the loop $g \mid \partial D$ is contractible in $Y-P(\Gamma)$.

To see this, consider the 2-manifold $U_{0}=U-\operatorname{Int} D$, with $\partial U_{0}=\partial D$. It is not hard to see that if we decompose $\bar{U}_{0}$ into the single points of $U_{0}$ and the components of $\bar{U}_{0}-U_{0}$, then the resulting decomposition space $Z$ is a disk. But each component of $\vec{U}_{0}-U_{0}$ is a connected subset of $g^{-1} P(A)$, and hence is mapped to a point by $g$. Thus, $g \mid \bar{U}_{0}$ can be expressed as the composition of the quotient map $\bar{U}_{0} \rightarrow Z$, and a map $Z \rightarrow Y-P(\Gamma)$. Hence, $g\left|\partial U_{0}=g\right| \partial D$ is an inessential map into $Y-P(\Gamma)$. The result follows.

Remark. The requirement in Theorem 4 that some polyhedral 3-manifold $H$ with free fundamental group should contain $A$, can be suppressed if we demand that $M^{3}$ be orientable (when $p>2$ ) and that $M^{3}$ contain no $Z_{p}$-homology 3-cells which fail to be simplyconnected. This follows from Corollary 2.1.

Recall our earlier convention and notation about compact decompositions.

Corollary 4.1. Let $M^{3}$ be a piecewise-linear 3-manifold, possibly with boundary, and let $G$ be a compact decomposition of $M^{3}$. Suppose that each element of $G$ is strongly 1-acyclic over $Z_{p}$ and has property 2UV. If $p>2$, assume also that $M^{3}$ is orientable. Then for each open, connected set $U \subset M^{3} / G$,

$$
P_{G} \mid P_{G}^{-1}(U): P_{G}^{-1}(U) \rightarrow U
$$

induces a monomorphism on fundamental groups.

Proof. Suppose $g: \Delta^{2} \rightarrow U$ and $f: \partial \Delta^{2} \rightarrow P_{G}^{-1}(U)$ are maps with $g \mid \partial \Delta^{2}=P_{G} f$. Let $F$ be the compact decomposition of $P_{G}^{-1}(U)$ associated with the compact set $\bar{H}_{G} \cap P_{G}^{-1} g\left(\Delta^{2}\right)$. An application to $P_{F}$ of Theorem 4 (and its remark) then yields the result.

Question. Does the conclusion of Corollary 4.1 follow if we retain the hypothesis that the elements of $G$ be strongly acyclic over $Z_{p}$ and have property 2-UV but, relax the requirement that the closure of $P_{G}\left(H_{G}\right)$ in $M^{3} / G$ be compact and 0 -dimensional?

Our next result is an improved version of [5, Lemma 1]. 
CoRollary 4.2. Let $F$ and $G$ be compact decompositions of $E^{3}$. Suppose that $F$ is strongly acyclic over $Z_{p}$ and that $h$ is an equivalence from $F$ to $G$. Let $d_{G}$ metrize $E^{3} / G$. Then for each positive $\epsilon$, there is a homeomorphism $R$ of $E^{3}$ onto $E^{3}$ such that $R$ agrees with $P_{G}^{-1} h P_{F}$ off the $\epsilon-$ neighborhood of $\bar{H}_{F}$, and

$$
d_{G}\left(P_{G} R(x), h P_{F}(x)\right)<\epsilon, \quad \text { for each } x \in E^{3} .
$$

In particular, if $h P_{F}(f)=P_{G}(g)$ for some $f \in F$ and $g \in G$, then $f$ is cellular if and only if $g$ is cellular.

Proof. Since $F$ is strongly acyclic over $Z_{p}, \bar{H}_{F}$ is definable by cubes-with-handles by Corollary 2.1. Thus, the $\epsilon$-neighborhood of $\bar{H}_{F}$ contains a compact polyhedron $L^{3}$ each of whose components is a cube-with-handles $\left(\bar{H}_{F} \subset \operatorname{Int} L^{3}\right)$, and such that $h P_{F}(K)$ has diameter less than $\epsilon$ for each component $K$ of $L^{3}$. It suffices to show that for each component $K$ of $L^{3}$, some homeomorphism of $K$ into the compact 3-manifold $K_{1}=P_{G}^{-1} h P_{F}(K)$ extends $P_{G}^{-1} h P_{F} \mid \partial K$.

The proof of J. Hempel's [13, Theorem 4] yields the fact that this extension is possible if and only if: For each polyhedral disk $D \subset K$, with $\partial D=D \cap \partial K$, it follows that $P_{G}^{-1} h P_{F} \mid \partial D$ represents a contractible loop in $K_{1}$. But this condition is met because of Corollary 4.1. The demonstration of our last claim about cellularity is routine, and is left to the reader.

Following M. L. Curtis in [11], we say that a separable metric $n$ dimensional space $Y$ is a homotopy $n$-manifold if each $y \in Y$ has arbitrarily small pairs of connected, open neighborhoods $V \subset U$ such that $\bar{V} \subset U, \bar{V}-V$ is connected, and the image of $\pi_{k}(V-y)$ in $\pi_{k}$ $(U-y)$ under the inclusion-induced homomorphism is isomorphic to $\pi_{k}\left(S^{n-1}\right)$ for each $k$. Curtis obtained the next result in the case when $G$ has only finitely many nondegenerate elements. In his situation, it is unnecessary to assume that $G$ is strongly 1 -acyclic.

Corollary 4.3. Let $G$ be a compact decomposition of $E^{3}$. Suppose that each element of $G$ is strongly 1-acyclic over $Z_{p}$. If $Y=E^{3} / G$ is a homotopy manifold, then each element of $G$ is cellular in $E^{3}$.

Proof. Since the proof is straightforward, we give only an outline. First of all, we use the existence, at each point of $Y$, of small neighborhoods with connected boundaries to show that each element of $G$ has property 2-UV (see Lemma 1, parts (iii) and (iv) and recall that the pre-image under $P_{G}$ of a compact and connected set is compact and connected).

Hence our Corollary 4.1 applies. It and the defining property (for 
$k=1$ ) of a homotopy 3-manifold can be used to verify the "cellularity criterion" hypothesis of [26, Corollary 3.2], which then gives our desired conclusion. Of course, the corollary quoted must be strengthened, using our present results, to cover the case $p>2$.

\section{REFERENCES}

1. S. Armentrout, Cellular decompositions of 3-manifolds that yield 3-manifolds, Bull. Amer. Math. Soc. 75 (1969), 453-456. MR 39 \#935.

2. - UV properties of compact sets, Trans. Amer. Math. Soc. 143 (1969), 487-498.

3. - Homotopy properties of decomposition spaces, Trans. Amer. Math. Soc. 143 (1969), 499-507.

4. - Shrinkability of certain decompositions of $E^{3}$ that yield $E^{3}$, Illinois J. Math. 13 (1969), 700-706.

5. S. Armentrout, L. L. Lininger and D. V. Meyer, Equivalent decomposition of $R^{3}$, Pacific J. Math. 24 (1968), 205-227. MR $36 \# 7117$.

6. S. Armentrout and T. M. Price, Decompositions into compact sets with UV properties, Trans. Amer. Math. Soc. 141 (1969), 433-442.

7. R. H. Bing, Conditions under which monotone decompositions of $E^{3}$ are simply connected, Bull. Amer. Math. Soc. 63 (1957), 143. Abstract \#325.

8. K. Borsuk, Theory of retracts, Monografie Mat., Tom. 44, PWN, Warsaw, 1967. MR $35 \# 7306$.

9. - Concerning homotopy properties of compacta, Fund. Math. 62 (1968), 223-254. MR $37 \# 4811$.

10. J. H. Case and R. E. Chamberlin, Characterizations of tree-like continua, Pacific J. Math. 10 (1960), 73-84. MR 22 \#1868.

11. M. L. Curtis, Shrinking continua in 3-space, Proc. Cambridge Philos. Soc. 57 (1961), 432-433. MR 22 \#11391.

12. W. Haken, Some results on surfaces in 3-manifolds, Studies in Modern Topology, Math. Assoc. Amer. (distributed by Prentice-Hall, Englewood Cliffs, N.J.), 1968, pp. 39-98. MR 36 \#118.

13. J. P. Hempel, $A$ surface in $S^{3}$ is tame if it can be deformed into each complementary domain, Trans. Amer. Math. Soc. 111 (1964), 273-287. MR 28 \#3409.

14. D. M. Hyman, ANR divisors and absolute neighborhood contractibility, Fund. Math. 62 (1968), 61-73. MR 37 \#4771.

15. V. P. Kompaniec and A. V. ČernavskiY, Equivalence of two classes of sphere mappings, Dokl. Akad. Nauk SSSR 169 (1966), 1266-1268=Soviet Math. Dokl. 7 (1966), 1083-1085. MR 39 \#2133.

16. G. Kozlowski, Factorization of certain maps up to homotopy, Proc. Amer. Math. Soc. 21 (1969), 88-92. MR 38 \#6588.

17. K. W. Kwun and F. Raymond, Almost acyclic maps of manifolds, Amer. J. Math. 86 (1964), 638-650. MR 32 \#1712.

18. R. C. Lacher, Cell-like mappings. I, Pacific J. Math. 30 (1969), 717-731.

19. - Cellularity criteria for maps, Michigan Math. J (to appear).

20. H. W. Lambert, Replacing certain maps of 3-manifolds by homeomorphisms, Proc. Amer. Math. Soc. 23 (1969), 676-678.

21. W. Magnus, A. Karrass and D. Solitar, Combinatorial group theory: Presentations of groups in terms of generators and relations, Pure and Appl. Math., vol. XIII, Interscience, New York, 1966. MR 34 \#7617. 
22. J. Martin, The sum of two crumpled cubes, Michigan Math J. 13 (1966), 147-151. MR 32 \#8324.

23. B. Mazur, A note on some contractible 4-manifolds, Ann. of Math. (2) 73 (1961), 221-228. MR 23 \#A2873.

24. D. R. McMillan, Jr., A criterion for cellularity in a manifold, Ann. of Math. (2) 79 (1964), 327-337. MR 28 \#4528.

25. - Strong homotopy equivalence of 3-manifolds, Bull. Amer. Math. Soc. 73 (1967), 718-722. MR 37 \#417.

26. —, Compact, acyclic subsets of three-manifolds, Michigan Math. J. 16 (1969), 129-136. MR 39 \#4822.

27. T. M. Ptice, $A$ necessary condition that a cellular upper semicontinuous decomposition of $E^{n}$ yield $E^{n}$, Trans, Amer. Math. Soc. 122 (1966), 427-435. MR 33 $\# 1843$.

28. L. C. Siebenmann, On detecting Euclidean space homotopically among topological manifolds, Invent. Math. 6 (1968), 245-261. MR 38 \#6601.

29. E. G. Skljarenko, Almost acyclic mappings, Mat. Sb. 75 (117) (1968), 296302 = Math. USSR Sb. 4 (1968), 267-272. MR 37 \#4806.

30. S. Smale, $A$ Vietoris mapping theorem for homotopy, Proc. Amer. Math. Soc. 8 (1957), 604-610. MR 19, 302.

31. E. H. Spanier, Algebraic topology, McGraw-Hill, New York, 1966. MR 35 $\# 1007$.

32. J. Stallings, Homology and central series of groups, J. Algebra 2 (1965), 170181. MR 31 \#232.

33. M. A. Stan'ko, Imbedding of tree-like compacta in $E^{3}$, Mat. Sb. 75 (117) (1968), 211-224 = Math. USSR Sb. 4 (1968), 191-202. MR 36 \#5918.

34. G. T. Whyburn, Compactness of certain mappings, Amer. J. Math. 81 (1959), 306-314. MR 22 \#1881.

35. A. Wright, Monotone mappings of compact 3-manifolds, Ph.D. Thesis, University of Wisconsin, Madison, 1969.

University of Wisconsin, Madison, Wisconsin 53706

Institute for Advanced Study, Princeton, New Jersey 08540 